\title{
ERRATUM
}

\section{Response to Belgard et al.}

E Skafidas, R Testa, D Zantomio, G Chana, IP Everall and C Pantelis

Molecular Psychiatry (2014) 19, 743-744; doi:10.1038/mp.2014.27; published online 18 March 2014

Correction to: Molecular Psychiatry (2014) 19, 407-409; doi:10.1038/mp.2013.186; published online 14 January 2014

Following publication of this paper, the authors noticed errors in Table 1. The gene number for SNP rs9832697 was missing; it should have been listed as 10 242. In addition, 107 SNPs were omitted. The corrected table appears in its entirety below:

\begin{tabular}{|c|c|c|c|c|c|}
\hline SNP & $\begin{array}{l}\text { Weight } \\
\text { lower }\end{array}$ & $\begin{array}{l}\text { Weight } \\
\text { median }\end{array}$ & $\begin{array}{c}\text { Weight } \\
\text { upper }\end{array}$ & $\begin{array}{c}\text { Gene } \\
\text { no. }\end{array}$ & $\begin{array}{c}\text { Gene } \\
\text { symbol }\end{array}$ \\
\hline rs968122 & 1.5465 & 1.5555 & 1.5645 & 27345 & KCNMB4 \\
\hline rs876619 & 0.9476 & 1.2092 & 1.4708 & 2775 & GNAO1 \\
\hline rs11020772 & 0.8553 & 0.8641 & 0.8729 & 2915 & GRM5 \\
\hline rs9288685 & 0.5856 & 0.5998 & 0.614 & 3635 & INPP5D \\
\hline rs10193128 & 0.5836 & 0.5946 & 0.6056 & 3635 & INPP5D \\
\hline rs7842798 & 0.5298 & 0.5386 & 0.5474 & 114 & $A D C Y 8$ \\
\hline rs3773540 & 0.5125 & 0.5208 & 0.5291 & 55799 & CACNA2D3 \\
\hline rs1818106 & 0.5002 & 0.5161 & 0.532 & 80310 & PDGFD \\
\hline rs2384061 & 0.4195 & 0.4306 & 0.4417 & 109 & $A D C Y 3$ \\
\hline rs12582971 & 0.3983 & 0.4295 & 0.4607 & 5288 & PIK3C2G \\
\hline rs10409541 & 0.4067 & 0.4189 & 0.4311 & 773 & CACNA1A \\
\hline rs2300497 & 0.3782 & 0.3889 & 0.3996 & 801 & CALM1 \\
\hline rs7562445 & 0.3741 & 0.3843 & 0.3945 & 2066 & ERBB4 \\
\hline rs7313997 & 0.3382 & 0.3567 & 0.3752 & 5801 & PTPRR \\
\hline rs2239118 & 0.3348 & 0.3552 & 0.3756 & 775 & CACNAIC \\
\hline rs4688054 & 0.1801 & 0.3476 & 0.515 & 2932 & GSK3B \\
\hline rs10823195 & 0.2597 & 0.3445 & 0.4294 & 1763 & DNA2 \\
\hline rs9798267 & 0.2759 & 0.3388 & 0.4017 & 84083 & ZRANB3 \\
\hline rs1075354 & 0.4236 & 0.3177 & 0.6402 & 55799 & CACNA2D3 \\
\hline rs1942052 & 0.2641 & 0.3088 & 0.3535 & 130013 & ACMSD \\
\hline rs4696443 & 0.2525 & 0.3047 & 0.3569 & 23321 & TRIM2 \\
\hline rs243196 & 0.2402 & 0.2976 & 0.3549 & 1112 & FOXN3 \\
\hline rs16929470 & 0.1854 & 0.2712 & 0.3571 & 775 & CACNA1C \\
\hline rs7580690 & 0.1647 & 0.2248 & 0.285 & 83439 & TCF7L1 \\
\hline rs7145618 & 0.1515 & 0.2238 & 0.296 & 5528 & $P P P 2 R 5 C$ \\
\hline rs3770132 & 0.1514 & 0.2093 & 0.2673 & 3676 & ITGA4 \\
\hline rs3790095 & 0.1215 & 0.2017 & 0.2819 & 2775 & GNAO1 \\
\hline rs1013459 & 0.1417 & 0.1969 & 0.2522 & 2774 & GNAL \\
\hline rs11001056 & 0.1519 & 0.1891 & 0.2263 & 5592 & PRKG1 \\
\hline rs10952662 & 0.148 & 0.1868 & 0.2257 & 26047 & CNTNAP2 \\
\hline rs7756516 & 0.152 & 0.1853 & 0.2186 & 3120 & HLA-DQB2 \\
\hline rs8054767 & 0.1322 & 0.1803 & 0.2284 & 5579 & PRKCB \\
\hline rs2239028 & 0.1121 & 0.1763 & 0.2405 & 775 & CACNA1C \\
\hline rs3935743 & 0.0969 & 0.1737 & 0.2505 & 5336 & PLCG2 \\
\hline rs4324526 & 0.1239 & 0.16 & 0.196 & 53343 & NUDT9 \\
\hline rs11736177 & 0.1047 & 0.1525 & 0.2004 & 5593 & PRKG2 \\
\hline rs3020827 & 0.1094 & 0.144 & 0.1786 & 3815 & KIT \\
\hline rs976266 & 0.0975 & 0.1373 & 0.1771 & 114 & $A D C Y 8$ \\
\hline rs884080 & 0.0999 & 0.136 & 0.172 & 5590 & PRKCZ \\
\hline rs7903424 & 0.0927 & 0.1319 & 0.1711 & 6934 & TCF7L 2 \\
\hline rs4465567 & 0.0837 & 0.1224 & 0.1611 & 10125 & RASGRP1 \\
\hline rs 2270838 & 0.059 & 0.1221 & 0.1852 & 11060 & WWP2 \\
\hline rs1029088 & 0.0662 & 0.1202 & 0.1741 & 6262 & RYR2 \\
\hline rs1873423 & 0.0555 & 0.1193 & 0.1831 & 5579 & PRKCB \\
\hline rs16931011 & 0.0708 & 0.1177 & 0.1646 & 3709 & ITPR2 \\
\hline rs2920022 & 0.0779 & 0.1164 & 0.1548 & 5908 & RAP1B \\
\hline rs10153211 & -0.0367 & 0.1154 & 0.2675 & 2903 & GRIN2A \\
\hline rs9469718 & 0.0241 & 0.1139 & 0.2037 & 2914 & GRM4 \\
\hline rs2036109 & 0.0782 & 0.1133 & 0.1484 & 148 & ADRA1A \\
\hline rs2684777 & 0.0727 & 0.1133 & 0.1539 & 3480 & IGF1R \\
\hline rs629720 & 0.065 & 0.1106 & 0.1562 & 80310 & PDGFD \\
\hline
\end{tabular}

\begin{tabular}{|c|c|c|c|c|c|}
\hline SNP & $\begin{array}{l}\text { Weight } \\
\text { lower }\end{array}$ & $\begin{array}{l}\text { Weight } \\
\text { median }\end{array}$ & $\begin{array}{c}\text { Weight } \\
\text { upper }\end{array}$ & $\begin{array}{c}\text { Gene } \\
\text { no. }\end{array}$ & $\begin{array}{c}\text { Gene } \\
\text { symbol }\end{array}$ \\
\hline rs831550 & 0.0734 & 0.1096 & 0.1458 & 1716 & DGUOK \\
\hline rs2394538 & 0.0242 & 0.1093 & 0.1945 & 3098 & HK1 \\
\hline rs948992 & 0.068 & 0.1068 & 0.1456 & 9965 & FGF19 \\
\hline rs2174257 & 0.0668 & 0.105 & 0.1432 & 5592 & PRKG1 \\
\hline rs11755661 & 0.0412 & 0.1041 & 0.167 & 5071 & PARK2 \\
\hline rs1928168 & 0.0657 & 0.099 & 0.1322 & 401237 & LINC00340 \\
\hline rs7100765 & 0.0434 & 0.0935 & 0.1436 & 5593 & PRKG2 \\
\hline rs1369450 & 0.0563 & 0.0924 & 0.1285 & 114 & $A D C Y 8$ \\
\hline rs1040336 & -0.0615 & 0.091 & 0.2435 & 2272 & FHIT \\
\hline rs10407144 & 0.0434 & 0.0872 & 0.131 & 773 & CACNA1A \\
\hline rs10794197 & 0.045 & 0.0869 & 0.1287 & 1488 & CTBP2 \\
\hline rs3734464 & 0.0247 & 0.0868 & 0.149 & 5071 & PARK2 \\
\hline rs7864216 & -0.0072 & 0.0863 & 0.1798 & 9630 & GNA14 \\
\hline rs4254056 & 0.0432 & 0.0846 & 0.126 & 338751 & OR52L1 \\
\hline rs988920 & 0.0453 & 0.0842 & 0.1232 & 9229 & DLGAP1 \\
\hline rs12393998 & 0.0536 & 0.0839 & 0.1142 & 8450 & CUL4B \\
\hline rs872794 & 0.0413 & 0.0813 & 0.1213 & 3778 & KCNMA1 \\
\hline rs2503220 & -0.0527 & 0.0806 & 0.214 & 5142 & PDE4B \\
\hline rs10468681 & 0.0356 & 0.08 & 0.1243 & 2774 & GNAL \\
\hline rs7258489 & 0.0428 & 0.079 & 0.1152 & 808 & CALM3 \\
\hline rs153968 & 0.0379 & 0.0765 & 0.115 & 5144 & PDE4D \\
\hline rs944761 & 0.0361 & 0.076 & 0.1159 & 9568 & GABBR2 \\
\hline rs2161630 & 0.0232 & 0.0754 & 0.1276 & 10725 & NFAT5 \\
\hline rs7097311 & 0.0294 & 0.0703 & 0.1111 & 5593 & PRKG2 \\
\hline rs2088747 & -0.0137 & 0.0693 & 0.1522 & 11060 & WWP2 \\
\hline rs9832697 & -0.0766 & 0.0689 & 0.2144 & 10242 & KCNMB2 \\
\hline rs7731023 & 0.0343 & 0.0683 & 0.1023 & 6502 & SKP2 \\
\hline rs7120612 & 0.0224 & 0.0659 & 0.1094 & 390055 & OR52A6 \\
\hline rs2033655 & 0.0277 & 0.0647 & 0.1017 & 109 & $A D C Y 3$ \\
\hline rs10768450 & 0.0261 & 0.0645 & 0.1029 & 119682 & OR51L1 \\
\hline rs3121309 & 0.018 & 0.0631 & 0.1083 & 89780 & WNT3A \\
\hline rs7067880 & 0.0035 & 0.0629 & 0.1222 & 5593 & PRKG2 \\
\hline rs2296328 & 0.0202 & 0.0614 & 0.1027 & 3710 & ITPR3 \\
\hline rs10208384 & 0.0154 & 0.0591 & 0.1027 & 55821 & ALLC \\
\hline rs5909975 & 0.029 & 0.0577 & 0.0864 & 2892 & GRIA3 \\
\hline rs10041275 & -0.003 & 0.0573 & 0.1177 & 2890 & GRIA1 \\
\hline rs2779531 & 0.0118 & 0.0559 & 0.1 & 9568 & $G A B B R 2$ \\
\hline rs1869901 & 0.0159 & 0.0539 & 0.0918 & 5330 & PLCB2 \\
\hline rs7809503 & 0.0119 & 0.0526 & 0.0932 & 51422 & PRKAG2 \\
\hline rs1881638 & 0.0125 & 0.0525 & 0.0924 & 51422 & PRKAG2 \\
\hline rs13359392 & -0.0036 & 0.0511 & 0.1058 & 2890 & GRIA1 \\
\hline rs2716191 & -0.0002 & 0.0466 & 0.0934 & 5608 & MAP2K6 \\
\hline rs11123677 & 0.0031 & 0.0462 & 0.0893 & 1261 & CNGA3 \\
\hline rs2937127 & -0.0358 & 0.0414 & 0.1187 & 11060 & WWP2 \\
\hline rs2305347 & -0.0055 & 0.0392 & 0.0839 & 23265 & EXOC7 \\
\hline rs2471226 & -0.0052 & 0.0389 & 0.083 & 107 & $A D C Y 1$ \\
\hline rs1881628 & -0.0107 & 0.0368 & 0.0843 & 51422 & PRKAG2 \\
\hline rs362249 & -0.0042 & 0.0361 & 0.0764 & 51807 & TUBA8 \\
\hline rs4708928 & -0.0031 & 0.036 & 0.0751 & 5071 & PARK2 \\
\hline rs1266348 & 0.0028 & 0.0359 & 0.0689 & 5634 & PRPS2 \\
\hline rs1186055 & -0.0032 & 0.0358 & 0.0747 & 5027 & $P 2 R X 7$ \\
\hline rs 1453541 & -0.1057 & 0.0354 & 0.1766 & 219983 & OR4D6 \\
\hline
\end{tabular}


Table. 1. (Continued)

\begin{tabular}{|c|c|c|c|c|c|}
\hline SNP & $\begin{array}{c}\text { Weight } \\
\text { lower }\end{array}$ & $\begin{array}{l}\text { Weight } \\
\text { median }\end{array}$ & $\begin{array}{c}\text { Weight } \\
\text { upper }\end{array}$ & $\begin{array}{c}\text { Gene } \\
\text { no. }\end{array}$ & $\begin{array}{c}\text { Gene } \\
\text { symbol }\end{array}$ \\
\hline rs3746821 & -0.0262 & 0.0335 & 0.0932 & 958 & CD40 \\
\hline rs220740 & -0.0085 & 0.0332 & 0.0749 & 10846 & PDE10A \\
\hline rs2299679 & -0.014 & 0.0331 & 0.0801 & 5332 & PLCB4 \\
\hline rs887387 & -0.0028 & 0.0317 & 0.0662 & 489 & ATP2A3 \\
\hline rs7174459 & -0.0092 & 0.0288 & 0.0669 & 4735 & NEDD5 \\
\hline rs884399 & -0.0073 & 0.0281 & 0.0634 & 5581 & PRKCE \\
\hline rs5021051 & -0.0146 & 0.027 & 0.0686 & 2895 & GRID2 \\
\hline rs2903813 & -0.0208 & 0.0252 & 0.0711 & 3315 & HSPB1 \\
\hline rs1062935 & -0.0207 & 0.0245 & 0.0697 & 57521 & RPTOR \\
\hline rs9347553 & -0.0154 & 0.0228 & 0.0609 & 5071 & PARK2 \\
\hline rs11072416 & -0.0259 & 0.0222 & 0.0703 & 6263 & RYR3 \\
\hline rs4553343 & -0.0304 & 0.0204 & 0.0712 & 2977 & GUCY1A2 \\
\hline rs7146234 & -0.0132 & 0.0202 & 0.0535 & 5495 & PPM1A \\
\hline rs848282 & -0.0191 & 0.0172 & 0.0536 & 55120 & FANCL \\
\hline rs7962764 & -0.0495 & 0.0126 & 0.0748 & 5801 & PTPRR \\
\hline rs12726519 & -0.0377 & 0.0098 & 0.0572 & 5321 & PLA2G4A \\
\hline rs718949 & -0.0303 & 0.0093 & 0.0489 & 1488 & CTBP2 \\
\hline rs1954787 & -0.0264 & 0.0089 & 0.0441 & 2900 & GRIK4 \\
\hline rs2238079 & -0.0283 & 0.0084 & 0.045 & 775 & CACNA1C \\
\hline rs1337420 & -0.0398 & 0.008 & 0.0558 & 2898 & GRIK2 \\
\hline rs917948 & -0.0553 & 0.0075 & 0.0704 & 5536 & РPР5C \\
\hline rs3817222 & -0.1848 & 0.0055 & 0.1957 & 4660 & PPP1R12B \\
\hline rs17531147 & -0.0612 & 0.003 & 0.0672 & 55970 & GNG12 \\
\hline rs6420481 & -0.0462 & 0.0014 & 0.0489 & 57521 & RPTOR \\
\hline rs38557 & -0.0428 & -0.0018 & 0.0392 & 781 & CACNA2D1 \\
\hline rs1430158 & -0.0542 & -0.0055 & 0.0432 & 5136 & PDE1A \\
\hline rs1480645 & -0.0542 & -0.0055 & 0.0432 & 27115 & PDE7B \\
\hline rs9173 & -0.0433 & -0.0061 & 0.0311 & 661 & POLR3D \\
\hline rs3924047 & -0.171 & -0.0072 & 0.1566 & 22941 & SHANK2 \\
\hline rs756944 & -0.0596 & -0.0077 & 0.0442 & 5608 & MAP2K6 \\
\hline rs2248068 & -0.0467 & -0.0099 & 0.0269 & 83439 & TCF7L1 \\
\hline rs12906456 & -0.0506 & -0.0116 & 0.0274 & 399694 & SHC4 \\
\hline rs6593958 & -0.0485 & -0.0121 & 0.0243 & 2005 & ELK4 \\
\hline rs4647992 & -0.0995 & -0.0145 & 0.0706 & 4790 & NFKB1 \\
\hline rs17193 & -0.0551 & -0.0149 & 0.0253 & 10846 & PDE10A \\
\hline rs702543 & -0.0508 & -0.0161 & 0.0186 & 5145 & PDE4D \\
\hline rs2283492 & -0.0595 & -0.0191 & 0.0213 & 115 & ADCY9 \\
\hline rs542413 & -0.0566 & -0.0201 & 0.0165 & 5581 & PRKCE \\
\hline rs731571 & -0.0608 & -0.0201 & 0.0207 & 4293 & МАРЗК9 \\
\hline rs2069812 & -0.0559 & -0.0201 & 0.0157 & 3567 & IL5 \\
\hline rs697851 & -0.0648 & -0.0215 & 0.0219 & 3708 & ITPKB \\
\hline rs933150 & -0.0947 & -0.0244 & 0.0459 & 326 & AIRE \\
\hline rs4748444 & -0.0611 & -0.0246 & 0.012 & 783 & CACNB2 \\
\hline rs7235163 & -0.0738 & -0.0375 & -0.0011 & 9229 & DLGAP1 \\
\hline rs4575213 & -0.0731 & -0.038 & -0.0029 & 5592 & PRKG1 \\
\hline rs11048476 & -0.0801 & -0.0384 & 0.0033 & 3709 & ITPR2 \\
\hline rs4145903 & -0.0762 & -0.0395 & -0.0028 & 783 & CACNB2 \\
\hline rs10505029 & -0.1011 & -0.0404 & 0.0203 & 51366 & UBR5 \\
\hline rs1122838 & -0.1213 & -0.0408 & 0.0396 & 9630 & GNA14 \\
\hline rs1993477 & -0.0818 & -0.0434 & -0.0049 & 51366 & UBR5 \\
\hline rs2179871 & -0.0912 & -0.0454 & 0.0005 & 10369 & CACNG2 \\
\hline rs10740244 & -0.0892 & -0.0467 & -0.0041 & 5592 & PRKG1 \\
\hline rs2503220 & -0.1151 & -0.0472 & 0.0207 & 5142 & PDE4B \\
\hline rs1065657 & -0.0838 & -0.0488 & -0.0139 & 51465 & UBE2J1 \\
\hline rs12714137 & -0.1234 & -0.0528 & 0.0179 & 83439 & TCF7L1 \\
\hline rs7176475 & -0.1275 & -0.0537 & 0.0201 & 123746 & PLA2G4E \\
\hline rs1937671 & -0.0953 & -0.0545 & -0.0138 & 5592 & PRKG1 \\
\hline rs7079293 & -0.0902 & -0.0549 & -0.0196 & 10581 & SORBS2 \\
\hline rs1003854 & -0.1288 & -0.0551 & 0.0187 & 326 & AIRE \\
\hline rs919741 & -0.0962 & -0.0565 & -0.0169 & 815 & CAMK2A \\
\hline rs750438 & -0.1075 & -0.0574 & -0.0074 & 11184 & MAP4K1 \\
\hline rs6139034 & -0.0997 & -0.0576 & -0.0154 & 3704 & ITPA \\
\hline rs1554606 & -0.1087 & -0.0599 & -0.0111 & 6018 & IL6 \\
\hline rs7108524 & -0.0938 & -0.0603 & -0.0267 & 81286 & OR51E3 \\
\hline rs1002424 & -0.1023 & -0.0626 & -0.0229 & 5562 & PRKAA1 \\
\hline rs2239316 & -0.1033 & -0.0631 & -0.0228 & 1387 & CREBBP \\
\hline rs5030949 & -0.157 & -0.0653 & 0.0264 & 3098 & HK1 \\
\hline rs17682073 & -0.1006 & -0.066 & -0.0315 & 6262 & $R Y R 2$ \\
\hline rs1872902 & -0.1108 & -0.0665 & -0.0221 & 80310 & PDGFD \\
\hline rs11602256 & -0.1035 & -0.0671 & -0.0308 & 4041 & LRP5 \\
\hline
\end{tabular}

Table. 1. (Continued)

\begin{tabular}{|c|c|c|c|c|c|}
\hline SNP & $\begin{array}{c}\text { Weight } \\
\text { lower }\end{array}$ & $\begin{array}{l}\text { Weight } \\
\text { median }\end{array}$ & $\begin{array}{l}\text { Weight } \\
\text { upper }\end{array}$ & $\begin{array}{c}\text { Gene } \\
\text { no. }\end{array}$ & $\begin{array}{c}\text { Gene } \\
\text { symbol }\end{array}$ \\
\hline rs10770675 & -0.1088 & -0.0675 & -0.0262 & 5139 & PDE3A \\
\hline rs9347587 & -0.1058 & -0.0696 & -0.0334 & 5071 & PARK2 \\
\hline rs4643498 & -0.1246 & -0.0778 & -0.0309 & 5136 & PDE1A \\
\hline rs 2451078 & -0.2263 & -0.0802 & 0.0659 & 93492 & TPTE2 \\
\hline rs3771832 & -0.2236 & -0.0803 & 0.063 & 6869 & TACR1 \\
\hline rs6508808 & -0.1506 & -0.0809 & -0.0112 & 6261 & RYR1 \\
\hline rs3748386 & -0.1475 & -0.0831 & -0.0187 & 11060 & WWP2 \\
\hline rs12207523 & -0.1622 & -0.0831 & -0.0041 & 5169 & ENPP3 \\
\hline rs2779556 & -0.1702 & -0.0867 & -0.0031 & 9568 & GABBR2 \\
\hline rs7918241 & -0.1351 & -0.0898 & -0.0444 & 1488 & CTBP2 \\
\hline rs2069837 & -0.1527 & -0.0924 & -0.0321 & 6018 & IL6 \\
\hline rs 12972670 & -0.1359 & -0.0926 & -0.0493 & 390892 & OR7A10 \\
\hline rs 2652452 & -0.1316 & -0.0989 & -0.0661 & 3760 & KCNJ3 \\
\hline rs9469529 & -0.1532 & -0.1004 & -0.0475 & 3710 & ITPR3 \\
\hline rs7677751 & -0.1496 & -0.1023 & -0.0551 & 5156 & PDGFRA \\
\hline rs7926083 & -0.1395 & -0.1028 & -0.0661 & 341276 & OR10A2 \\
\hline rs12577638 & -0.1598 & -0.11 & -0.0601 & 2900 & GRIK4 \\
\hline rs 11102321 & -0.1838 & -0.1104 & -0.037 & 5906 & RAP1A \\
\hline rs 1050395 & -0.1576 & -0.1154 & -0.0732 & 490 & ATP2B1 \\
\hline rs8103699 & -0.1586 & -0.1196 & -0.0805 & 773 & CACNA1A \\
\hline rs7971175 & -0.1566 & -0.1201 & -0.0836 & 5502 & PPPIR1A \\
\hline rs11602535 & -0.166 & -0.1236 & -0.0812 & 219981 & OR5A2 \\
\hline rs11644436 & -0.1733 & -0.1253 & -0.0774 & 5336 & PLCG2 \\
\hline rs 10762342 & -0.1909 & -0.1283 & -0.0658 & 5592 & PRKG1 \\
\hline rs11583646 & -0.2023 & -0.1311 & -0.0599 & 6262 & RYR2 \\
\hline rs6118611 & -0.1819 & -0.1321 & -0.0822 & 5332 & PLCB4 \\
\hline rs2587891 & -0.1722 & -0.1322 & -0.0922 & 2775 & GNA01 \\
\hline rs4651343 & -0.1739 & -0.1333 & -0.0926 & 5321 & PLA2G4A \\
\hline rs1659506 & -0.1761 & -0.1363 & -0.0966 & 23295 & MGRN1 \\
\hline rs2271986 & -0.1968 & -0.1367 & -0.0767 & 4842 & NOS1 \\
\hline rs2302898 & -0.1775 & -0.1375 & -0.0975 & 10381 & TUBB3 \\
\hline rs6971999 & -0.2088 & -0.1425 & -0.0763 & 26212 & OR2F2 \\
\hline rs2272197 & -0.1896 & -0.1485 & -0.1073 & 4216 & MAP3K4 \\
\hline rs4947963 & -0.1867 & -0.1493 & -0.1119 & 1956 & $E G F R$ \\
\hline rs7536307 & -0.1876 & -0.1507 & -0.1138 & 26289 & $A K 5$ \\
\hline rs12462609 & -0.2085 & -0.151 & -0.0936 & 773 & CACNA1A \\
\hline rs1517521 & -0.2925 & -0.152 & -0.0114 & 23180 & RFTN1 \\
\hline rs8063461 & -0.1865 & -0.1534 & -0.1203 & 7249 & TSC2 \\
\hline rs888817 & -0.1937 & -0.1604 & -0.1272 & 5924 & RASGRF2 \\
\hline rs922445 & -0.2435 & -0.1659 & -0.0883 & 2775 & GNAO1 \\
\hline rs339408 & -0.203 & -0.167 & -0.131 & 9322 & TRIP10 \\
\hline rs 7512378 & -0.2068 & -0.1691 & -0.1314 & 55811 & $A D C Y 10$ \\
\hline rs7870040 & -0.2408 & -0.1892 & -0.1376 & 774 & CACNA1B \\
\hline rs3904668 & -0.2423 & -0.2069 & -0.1715 & 29993 & PACS1N1 \\
\hline rs12716928 & -0.2784 & -0.2073 & -0.1362 & 5336 & PLCG2 \\
\hline rs6679454 & -0.262 & -0.2305 & -0.199 & 1601 & $D A B 2$ \\
\hline rs 10783235 & -0.2951 & -0.2549 & -0.2147 & 121275 & OR10AD1 \\
\hline rs3910363 & -0.3063 & -0.2611 & -0.2159 & 57495 & K1AA1239 \\
\hline rs 16853387 & -0.3799 & -0.3367 & -0.2935 & 26154 & $A B C A 12$ \\
\hline rs 17629494 & -0.5242 & -0.507 & -0.4898 & 5592 & PRKG1 \\
\hline rs4648135 & -0.5807 & -0.526 & -0.4713 & 4790 & NFKB1 \\
\hline rs 17643974 & -0.5527 & -0.5424 & -0.5321 & 1488 & CTBP2 \\
\hline rs1243679 & -0.5771 & -0.5674 & -0.5577 & 341799 & OR6S1 \\
\hline rs2240228 & -0.5942 & -0.5816 & -0.569 & 26532 & $\mathrm{OR} 10 \mathrm{H} 3$ \\
\hline rs260808 & -0.5938 & -0.5836 & -0.5734 & 80310 & PDGFD \\
\hline rs4128941 & -0.6166 & -0.6082 & -0.5998 & 8313 & AXIN2 \\
\hline rs769052 & -0.6321 & -0.6235 & -0.6149 & 7322 & UBE2D2 \\
\hline rs984371 & -0.7273 & -0.7181 & -0.7089 & 219437 & ORSL1 \\
\hline rs4308342 & -1.0196 & -0.8938 & -0.768 & 1633 & $D C K$ \\
\hline rs11145506 & -0.94 & -0.9172 & -0.8944 & 9630 & GNA14 \\
\hline rs905646 & -0.97 & -0.9624 & -0.9548 & 2915 & GRM5 \\
\hline rs6483362 & -0.9894 & -0.9661 & -0.9428 & 2915 & GRM5 \\
\hline rs 12317962 & -1.4869 & -1.32 & -1.1531 & 27345 & KCNMB4 \\
\hline rs8053370 & -1.7162 & -1.6956 & -1.675 & 2775 & GNAO1 \\
\hline
\end{tabular}

Abbreviations: ASD, autism spectrum disorder; CEU, Central European; SNP, single-nucleotide polymorphism. Weight indicates the contribution of each SNP to ASD clinical status. The lower and upper weights represent the $95 \%$ confidence intervals $(\mathrm{Cls})$ of the distribution of weights for each SNP. 